\title{
Incidence of Anxiety in Patients with Multiple Sclerosis (MS)
}

\author{
Rivka Green ${ }^{1, *}$, Jennifer Kalina ${ }^{2}$ and Krupa Pandey ${ }^{1}$ \\ ${ }^{1}$ Multiple Sclerosis Comprehensive Care Center, Barnabas Health 200 South Orange Ave., Suite 124-A, \\ Livingston, NJ 07039, USA \\ ${ }^{2}$ Multiple Sclerosis Comprehensive Care Center, NYU Langone Medical Center, 240 East 38th St., 18th \\ Floor, New York, NY 10016, USA
}

\begin{abstract}
Objective: To investigate the (1) incidence of anxiety and (2) association of anxiety with disease duration, depression, and age in an outpatient Multiple Sclerosis (MS) Center.

Background: The incidence of anxiety varies in the literature but is estimated to affect $44.5 \%$ of people with MS. Anxiety can be a predictor of poor Quality of Life, especially relevant in progressive illnesses, such as MS. Though research has shown that patients newly diagnosed experience higher anxiety levels, the relationship between anxiety and disease duration is unclear. Since anxiety can be comorbid with depression in MS populations, it is relevant to establish its association in this setting. Finally, though anxiety usually increases in older age, research for this relationship in MS populations is inconclusive.

Design/Methods: The commonly used Hospital Anxiety and Depression Scale, was distributed once to patients with MS over a 3 month period to assess anxiety and depression scores. MS disease duration and age were confirmed by neurologists.

Results: Out of 160 patients with MS who completed the HADS, 19\% reported abnormal anxiety, $14 \%$ reported borderline cases of anxiety, and $67 \%$ did not report anxiety. After following up on significant correlations, depression and younger age were related to higher anxiety levels, $(R 2=.35, F(4,144)=19.26, p<0.001)$. The standardized partial regression coefficients indicated 2 statistically significant predictors, depression $(\beta=.55, p=<.001)$ and age $(\beta=-.25$, $p=<.001)$.

Discussion: $33 \%$ of patients reported symptoms of anxiety, emphasizing the need to focus on treating anxiety, specifically, early in their disease course. Clinicians may also want to recognize that elevated depression scores predicted anxiety. Lastly, in our cohort, the younger population reported higher anxiety, demonstrating that progressive illnesses may affect anxiety levels in younger populations more. Since approximately one third of patients with MS expressed anxiety symptoms, it is important to incorporate this into treatment plans.
\end{abstract}

Keywords: Multiple Sclerosis (MS), anxiety, disease duration, age, disability.

\section{INTRODUCTION}

Multiple Sclerosis (MS) is often a progressive demyelinating disorder that affects approximately 2.5 million people worldwide and 400,000 people in the US [1]. It is the most common cause of neurological disability in adults, both young and middle aged [2-4]. MS is usually diagnosed in persons between the ages of 20 to 40 with an unpredictable disease course [5]. MS presents with a wide range of symptoms including gait abnormalities, sensory disturbances, and memory dysfunction, along with emotional manifestations such as depression and anxiety $[1,2,6]$.

In general, anxiety and depression often present together [7]. Anxiety can be defined as an emotion that is characterized by feelings of tension, worry and physical changes [8]. Depression can be defined as experiencing lack of interest and pleasure, weight gain

*Address correspondence to this author at the Multiple Sclerosis Comprehensive Care Center, Barnabas Health 200 South Orange Ave., Suite 124-A, Livingston, NJ 07039, USA; Tel: 973-322-7073; Fax: 973-322-7802; E-mail: RGreen@barnabashealth.org or loss, differences in sleep patterns, lack of concentration and feelings of worthlessness or guilt [8]. Depression may also include thoughts of death or suicide. In MS populations, depression is prevalent in approximately $50 \%$ of the MS population [2], and often may occur with anxiety [6, 9-10]. Independently, anxiety is found in approximately $40 \%$ of the MS population [3, 11]. While numerous studies have analyzed many of these MS symptoms including depression in MS populations, anxiety has received far less attention [2, 6, 9, 10]. In a study reviewing standardized measures of depression and anxiety, only 5 out of the 15 studies had standardized measures of anxiety, while measures of depression were assessed in all 15 [12]. Although anxiety was far less frequently assessed than depression, research has suggested that the rates of anxiety may be twice as common as depression in MS populations [10]. Therefore, researching the incidence of anxiety and its predictors can benefit clinicians working with this population.

Previous research has estimated that persons with MS report significantly higher rates of anxiety as compared to the general population [3, 4, 13-15]. In MS 
populations, anxiety has been linked to social dysfunction and suicidal ideation [2, 10]. Anxiety is also of major concern in MS because anxiety can involve somatic complaints such as unsteadiness or dizziness, which are also common symptoms in MS. These symptoms may or may not be related to the anxiety thereby making it difficult to differentiate the etiology of these symptoms.

Current standard of care treatments for MS focuses on improving Quality of Life (QOL) by delaying disease progression and symptomatic relief. By researching the incidence of anxiety and its correlates, we may have a better understanding of how symptoms of anxiety affect people with MS. Studies on MS and anxiety have looked at correlates of anxiety in MS populations, including depression, fatigue, cognitive impairment, disability status, and time of diagnosis [4, 5, 10, 14-16]. The current study analyzes the following correlates with anxiety including, disease duration, depression and age, while controlling for disability. This ensures that disability status is not influencing the rates of anxiety.

At diagnosis, patients with MS are faced with enormous uncertainty and unpredictability [17]. Feelings of uncertainty may disrupt psychological wellbeing and contribute to anxiety disorders [17]. This is why many newly diagnosed patients with MS experience elevated anxiety levels [17]. Studies have shown that anxiety levels decrease after the first year of diagnosis [17]. Whether or not anxiety levels remain high or become lower throughout disease duration varies in the literature. Some studies, using the Hospital Anxiety and Depression Scale (HADS) to screen for anxiety, found small inverse correlations between anxiety and disease duration [18]. Additionally, in longitudinal studies, levels of anxiety have been seen to decrease overtime from diagnosis [10]. This means that as the disease course continues, anxiety levels may decrease. Other studies, however, did not find any relationship between disease duration and anxiety [4, 15]. The current study measures the relationship between disease duration and anxiety in our outpatient center to determine if there is any significant association.

Most studies in MS have found a positive association between anxiety and depression, similar to the general population, and indicating that these 2 conditions may be associated with one another [10, 18]. Moreover, Garfield's study [14] showed a positive association between anxiety and depression levels, specifically using the HADS to screen for anxiety and depression. Further, Wood's study [10] demonstrated that depression was unlikely to occur alone, and is often only accompanied by anxiety or fatigue in MS populations. Other studies have found that besides gender, depression was the only predictor of anxiety in MS using the HADS [14, 19-21]. Most studies seem to have found a positive correlation between anxiety and depression in MS populations $[13,18]$.

As compared to anxiety and depression, the relationship between anxiety and age in persons with MS varies in the literature. Some larger studies, using the HADS to screen for anxiety, did not find a correlation between anxiety and age $[4,18]$. However, other studies have found an association between lower anxiety levels with older age [10] and higher anxiety levels with younger age [15]. This suggests that in MS populations, anxiety may decrease with age. Researchers have proposed that the reason for this may be that as one ages, treatment options have been established, social support structures may have developed and acceptance of MS may have occurred, thereby, altering one's lifestyle to be more positive [10]. Generally, anxiety levels increase with age in the general population. However, in MS populations anxiety levels may be increased with younger people who are faced with a diagnosis and harder treatment decisions because of the long disease duration. The current study examines if age can influence anxiety levels in MS populations.

More severe disease disability has been shown to be associated with depression but the association between disability and anxiety varies in the literature [5]. Many studies in this area have used the Expanded Disability Status Scale (EDSS), an objective measure of disability that is performed by a neurologist to track disability in MS populations [10]. Many studies, using the EDSS, have found no association between disability and anxiety reports $[2,4,6]$. In a longitudinal study, however, Woods' study [10] did find higher EDSS scores to be associated with higher anxiety at cohort entry, but not related overtime. Interestingly, Nicholl's study [12], which used the Guy's Neurological Disability Scale (GNDS) did find an association between disability and anxiety. A different scale, entitled Patient Determined Disease Steps (PDDS) [22] was used in the current study and measures disability. The current study controls for disability status to ensure that it does not affect the variance in anxiety scores.

Due to the high prevalence of anxiety in MS populations and the severe debilitation that MS can 
cause, our study aims to add to the knowledge of anxiety and MS and determine if disease duration, depression or age are associated with anxiety scores in an outpatient suburban MS center.

\section{METHODS}

\section{Participants}

The present study took place at the Barnabas Health Multiple Sclerosis Comprehensive Care Center and was approved by the Saint Barnabas Medical Center Medical Education and Clinical Research Committee. Patients were between the ages of 22 and 71 with an average age of $45.81(S D=0.87)$. Out of 160 patients who participated in the study, $86 \% \quad(n=137)$ were female. The mean disease duration was 11 years $(S D=0.60)$. Eighty-two percent $(n=131)$ had a diagnosis of RRMS, $14 \%(n=23)$ had a diagnosis of SPMS, and $4 \%(n=6)$ had a diagnosis of PPMS. Patients were included if they had an MS diagnosis, had the ability to understand English, and were seen at the MS Center for follow up routine neurological visits.

\section{Procedure}

During routine appointments to the MS Center, patients filled out the HADS and the PDDS as routine standard of clinical care visits. One hundred and sixty patients with MS completed the HADS for anxiety and depression. Thirteen were removed due to incomplete questionnaires, defined as missing 1 or more questions. Demographic variables, such as race, gender, and age were retrospectively collected using the patients' medical records. The neurologists determined disease duration.

\section{Measures}

The HADS was used to assess anxiety and depression. The HADS is a 14-item self-report instrument designed to screen for anxiety and depression in hospital settings. Each item is rated on a 0 ("only occasionally") to a 3 ("a great deal of the time") point scoring level of agreement with the statement. Example of statements include: "Worrying thoughts go through my mind". A total score is given independently for the subscales: 1) anxiety and 2) depression and the score for each subscale can range from 0 to 21 . Total scores are calculated by adding the individual score for each question. Scores between 0-7 indicate a "normal case" or unlikely depression or anxiety, scores between 8-9 indicate a "borderline case" or indicative of possible depression or anxiety, and scores above 10 indicate an "abnormal case" or reflecting probable anxiety or depression [9].

Although there are numerous self-report scales that are used to detect anxiety in medical populations, the HADS has been validated for use in MS populations [9], having good internal reliability in MS, with Chronbach alpha-values for anxiety, depression and total scores at $0.83,0.77,0.87$ [13]. The HADS is appropriate as an anxiety screening in MS because it excludes somatic items, not allowing for patients to confuse those symptoms with anxiety [6].

The PDDS is a patient derived, self-reported scale that measures neurological impairment. The PDDS is ranked on a $0-8$ point scale where 0 indicates no disability from MS (E.g. "I may have some mild symptoms, mostly sensory due to MS but they do not limit my activity. If I do have an attack, I return to normal when the attack has passed") and 8 indicates bedridden due to MS (E.g. "Unable to sit in a wheelchair for more than one hour"). Higher scores on the PDDS indicate a greater disability status. The PDDS has been strongly related to EDSS scores $(r=0.87)[22]$.

\section{RESULTS}

Out of 160 patients with MS who completed the anxiety portion of the HADS, $19 \%$ reported abnormal anxiety (score $=\geq 10$ ), $14 \%$ reported borderline cases of anxiety (score $=8-9$ ), and $67 \%$ did not report anxiety (score =0-7). The mean anxiety score was 6.29 (SD = 4.36). PDDS scores ranged from 0 to 7 with a mean of $2.09(S D=0.16)$.

One hundred and fifty seven patients completed the depression portion of the HADS and $8.4 \%$ reported abnormal depression (score $=\geq 10$ ), $10.1 \%$ reported borderline cases of depression (score $=8-9$ ), and $81.5 \%$ did not report depression (score $=0-7$ ). The mean depression score was $4.22(S D=3.46)$.

A Pearson correlation was preformed to determine whether there was a relationship between disease duration, depression and age with anxiety. Firstly, disease duration was not significantly related to anxiety $(r=-.14, p=.08)$. Secondly, anxiety and depression scores were positively correlated $(r=0.52, p<.001)$. Lastly, age was negatively related to anxiety $(r=-0.24$, $p<.001)$. PDDS scores were not associated with anxiety scores. 
A multiple hierarchal regression was performed to see if disease duration, depression and age could predict anxiety after controlling for disability status. Disease disability was entered first step, followed by disease duration, depression, and age in the second step.

Disease disability was not significantly related to the outcome and therefore, it did not confound the results of the regression. The control predictor variables, depression and age, were significantly related to this outcome, $\quad\left(R^{2}=.35, \quad F(4,144)=19.26, \quad p<0.001\right.$ ) (see Table 1). Depression and age explained $19 \%$ of the variance. The standardized partial regression coefficients indicated 2 statistically significant predictors, depression $(\beta=.55, p=<.001)$ and age $(\beta=-$ $.25, p=<.001$ ).

Table 1: Summary of Hierarchical Regression for Disease Duration, Depression and Age on Predicting Anxiety ( $\mathrm{N}=149)$

\begin{tabular}{|c|c|c|c|}
\hline Variable & $\boldsymbol{B}$ & SE $^{\mathbf{b}}$ & $\boldsymbol{\beta}$ \\
\hline \hline Step 1 & .173 & 0.48 \\
\hline Disease disability & .102 &. \\
\hline Step 2 & .044 & -.061 \\
\hline Disease duration & -.036 & .090 & $.551^{*}$ \\
\hline Depression & .696 & .031 & $-.247^{*}$ \\
\hline Age & -.096 &
\end{tabular}

Note: $\mathrm{R} 2=0.02$ for step $1 ; \Delta \mathrm{R} 2=0.330$ and $\mathrm{F}$ change $=19.26, p=0.00$ for step 2.

${ }^{*} p<0.005$.

\section{DISCUSSION}

A third of the MS patients in our sample reported more than average anxiety levels on the HADS. This is consistent with previous research that indicates that anxiety levels are higher in MS populations than the general public $[3,4,13-15]$. This percentage is also consistent with previous research using the HADS that reports anxiety levels to be between $25-41 \%[3,18]$.

This study used a patient reported PDDS and found that perceptions of disability are not related to reports of anxiety levels. This means that although patients may perceive their disease as less or more severe, it does not relate to how they report anxious symptoms. An interesting point about the PDDS is that currently minimal research exists on patients' subjective perception of their disease, which is captured by the PDDS. Most studies in MS use the EDSS to measure neurological disability.
Although the relationship between anxiety and disease duration varies in the literature $[4,10,13-15$, $17,18]$, our study found a weak negative relationship between the two, indicating that anxiety levels may be highest in the earlier stages of one's disease course. However, disease duration did not account for variance in anxiety scores in the regression analysis. Since an inverse relationship exists, there is a need for empathy and sensitivity early on in a patient's disease course. It seems that even after the initial shock of diagnosis passes, anxiety levels may remain high in the beginning of one's disease course. Further research may want to investigate this relationship further with a larger sample to validate these findings.

Results of the regression indicated that both higher depression scores and younger age significantly and independently predicted anxiety in this population, controlling for the effects of disability in this regression model. This means that high depression scores are a key predictor of higher anxiety levels. It is important for clinicians, on recognizing the presence of depression, to have awareness of the likely presence of anxiety, specifically in MS populations. More importantly, screening for depression as a comorbid condition at the time of diagnosis may allow for better treatment.

Finally, younger age predicted anxiety levels indicating that younger people with MS had greater risk of anxiety. In accordance with previous research, this may be because initially, patients have not developed adequate social support mechanisms and coping methods useful for mitigating anxiety [10]. Moreover, we suggest that when one is faced with a progressive disease at a young age, one may be anxious to the limitations and restrictions that he or she has at a younger age.

\section{FUTURE WORK}

Treatment for anxiety in MS is under researched [15] and while the present study recognizes that anxiety is prevalent, future studies should focus on successful interventions and implementation techniques specific to MS populations. Although selfreport measures are useful and cost effective, they cannot alone classify symptomologies. This study relied on self-reported information and results may have inherent biases, yet they still may be useful as screening instruments. Screening for these symptoms as a comorbid condition rather than a result of the diagnosis is important prior to diagnosis being given. As well, exposure to psychologists or psychiatric treatment was not controlled for, so anxiety levels may 
have been further reduced in these people, confounding the results.

Similar to prior research in MS populations, there was an over representation of women in our sample and this reflects MS populations. Further work should attempt to screen an equal number of patients in each gender category since this may have biased our results.

\section{CONCLUSIONS}

A third of the MS patients in our sample reported higher than average anxiety levels on the HADS. The most significant predictors of anxiety in our cohort were higher depression scores and younger age. The current study suggests that anxiety levels should be assessed, especially at earlier stages of MS and younger ages. MS is the most common neurological disease among young adults [23]. The significance of anxiety treatment and care has major potential to improve quality of life for persons with MS and therefore, it is important for clinicians to assess and manage anxiety levels from time of diagnosis [18].

\section{REFERENCES}

[1] Multiple Sclerosis [Internet] 2014. Available from: www.clevelandclinicmeded.com/medicalpubs/diseasemanageme nt/neurology/multiple_sclerosis/ [accessed on 12 December 2014].

[2] Feinstein A, O'Connor P, Gray T, Feinstein K. The effects of anxiety on psychiatric morbidity in patients with multiple sclerosis. Mult Scler 2000; 5: 323-6.

\section{http://dx.doi.org/10.1177/135245859900500504}

[3] Korostil M, Feinstein A. Anxiety disorders and their clinical correlates in multiple sclerosis patients. Mult Scler 2007; 13(1): 67-72.

http://dx.doi.org/10.1177/1352458506071161

[4] Dahl OP, Stordal E, Lydersen S, Midard R. Anxiety and depression in multiple sclerosis. A comparative population-based study in Nord-Trondelag County, Norway. Mult Scler 2009; 15(12): 1495-1501.

http://dx.doi.org/10.1177/1352458509351542

[5] Vuger-Kovacic D, Gregurek R, Kovacic D, Vuger T, Kalenic, B. Relation between anxiety, depression and locus of control of patients with multiple sclerosis. Mult Scler 2007; 13(8): 1065-7. http://dx.doi.org/10.1177/1352458507077629

[6] Donnchadha SO, Burke T, Bramham J, et al. Symptom overlap in anxiety and multiple sclerosis. Mult Scler 2013; 19(10): 1349-54. http://dx.doi.org/10.1177/1352458513476742

[7] Anxiety and Depression Association of America (ADAA) Treatment [Internet] 2015. Available from: http://www.adaa. org/understanding-anxiety/depression/treatment. [accessed on 25 March 2015].
[8] Kazdin AE. Encyclopedia of Psychology. New York, NY: Oxford Univeristy Press 2000.

[9] Honarmand K, Feinstein A. Validation of the Hospital Anxiety and Depression Scale for use with multiple sclerosis patients. Mult Scler 2009; 15(12): 1518-24. http://dx.doi.org/10.1177/1352458509347150

[10] Wood B, van der Mei IAF, Ponsonby AL, et al. Prevalence and concurrence of anxiety, depression and fatigue over time in multiple sclerosis. Mult Scler 2013; 19(2): 217-24. http://dx.doi.org/10.1177/1352458512450351

[11] Galeazzi GM, Ferrari S, Giaroli G, et al. Psychiatric disorders and depression in multiple sclerosis outpatients: impact of disability and interferon beta therapy. Neurol Sci 2005; 26: 255-62. http://dx.doi.org/10.1007/s10072-005-0468-8

[12] Nicholl CR, Lincoln NB, Francis VM, et al. Assessment of emotional problems in people with multiple sclerosis. Clin Rehabil 2001; 15: 657-68 http://dx.doi.org/10.1191/0269215501cr427oa

[13] Atkins L, Newby G, Primm J. Normative Data for the Hospital Anxiety and Depression Scales (HADS) in multiple sclerosis. Soc Care Neurodis 2012; 3(4): 172-8. http://dx.doi.org/10.1108/20420911211286579

[14] Garfield AC, Lincoln NB. Factors affecting anxiety in multiple sclerosis. Disabil Rehabil 2012; 34(24): 2047-52. http://dx.doi.org/10.3109/09638288.2012.667503

[15] Beiske AG, Svensson E, Sandanger B, et al. Depression and anxiety amongst multiple sclerosis patients. Eur J Neurol 2008; 15: 239-245. doi: 10.1111/f.1468-1331.2007.02041x

[16] Lester K, Stepleman L, Hughes M. The association of illness severity, self-reported cognitive impairment, and perceived illness management with depression and anxiety in a multiple sclerosis clinic population. J Behav Med 2007; 30(2): 177-86. http://dx.doi.org/10.1007/s10865-007-9095-6

[17] Janssens ACJW, van Doorn PA, de Boer JB, van der Meche FGA, Passchier J, Hintzen RQ. Impact of recently diagnosed multiple sclerosis on quality of life, anxiety, depression, and distress of patients and partners. Acta Neurol Scandinavica 2003; 108: 389-95.

http://dx.doi.org/10.1034/j.1600-0404.2003.00166.x

[18] Jones KH, Ford DV, Jones PA, et al. A Large-Scale Study of Anxiety and Depression in People with Multiple Sclerosis: A Survey via the Web Portal of the UK MS Register. PloS One 2012; 7(7): e41910.

http://dx.doi.org/10.1371/journal.pone.0041910

[19] Brown RF, Valpiani EM, Tennant CC, et al. Longitudinal assessment of anxiety, depression, and fatigue in people with multiple sclerosis. Psychol Psychother-T 2009; 82(1): 41-56. http://dx.doi.org/10.1348/147608308X345614

[20] Giordano A, Granella F, Lugaresi A, et al. Anxiety and depression in multiple sclerosis patients around diagnosis. $J$ Neurol Sci 2011; 307(1-2): 86-91. http://dx.doi.org/10.1016/i.jns.2011.05.008

[21] Hartoonian N, Beier ML, Turner AP, Day MA, Alschuler KN. Predictors of Anxiety in Multiple Sclerosis. Rehabil Psychol 2014, 60(1): 91-8.

http://dx.doi.org/10.1037/rep0000019

[22] Learmonth YC, Motl RW, Sandroff BM, et al. Validation of patient determined disease steps (PDDS) scale score in persons with multiple sclerosis. BMC Neurol 2013; 13: 37-44. http://dx.doi.org/10.1186/1471-2377-13-37

[23] National Multiple Sclerosis Society (NMSS) What is MS? [Internet] 2015. Available from: http://www.nationalmssociety. org/What-is-MS. [accessed on 14 April 2015].

\section{DOI: http://dx.doi.org/10.6000/2292-2598.2015.03.02.6}

(C) 2015 Green et al.; Licensee Lifescience Global.

This is an open access article licensed under the terms of the Creative Commons Attribution Non-Commercial License (http://creativecommons.org/licenses/by-nc/3.0/) which permits unrestricted, non-commercial use, distribution and reproduction in any medium, provided the work is properly cited. 